\title{
Percepção de estudantes sobre PBL Online em cursos superiores do Instituto Federal da Bahia durante a pandemia da Covid-19
}

\author{
Davy M. S. Gomes ${ }^{1}$, Luciano T. Silva ${ }^{1}$, Rebeca dos Santos ${ }^{2}$, Fernanda Silveira ${ }^{2}$, \\ Fernanda C. B. Santana ${ }^{1}$, Igo Amauri S. Luz ${ }^{2}$ \\ ${ }^{1}$ Área de Informática - Instituto Federal da Bahia (IFBA), \\ Campus Feira de Santana, BR-324 - Aviário, Feira de Santana - BA - Brasil \\ ${ }^{2}$ Instituto Federal da Bahia (IFBA), Campus Santo Antônio de Jesus \\ \{davymatos01, teleslu9, beekahjesus18, nandasilva36m\}@gmail.com \\ \{fernandacastelo, igo.luz\}@ifba.edu.br
}

\begin{abstract}
In March 2020, the World Health Organization classified the outbreak of contamination by the new coronavirus as a pandemic. From the declaration, activities considered non-essential were suspended worldwide. One of the areas that has been impacted is education - with the closure of schools and universities, many institutions needed to innovate in their practices by implementing remote education and the Federal Institute of Bahia (IFBA) did so through Emergency Non-Presential Teaching Activities that were regulated through its own resolution. We aimed to present the perception of students of IFBA higher education courses in the computing area who participated in curricular and extracurricular activities performed from the application of the Problem-Based Learning (PBL) methodology, an active methodology that we have adapted to be applied in an online environment. Student feedback regarding essential aspects of the experience will be presented, especially that related to the construction of the problem, execution infrastructure and competences and skills that have been developed.
\end{abstract}

Resumo. Em março de 2020, a Organização Mundial de Saúde classificou o surto de contaminação pelo novo coronavírus como uma pandemia e, a partir da declaração, atividades consideradas não essenciais foram suspensas em todo o mundo. Uma das áreas que foi impactada foi a educação - com o fechamento das escolas e universidades, muitas instituições necessitaram inovar em suas práticas implantando o ensino remoto e o Instituto Federal da Bahia (IFBA) assim ofez através das Atividades De Ensino Não Presenciais Emergenciais, regulamentadas através de resolução própria. O objetivo deste artigo é apresentar a percepção dos estudantes de cursos superiores da área de computação do IFBA que participaram de atividades curriculares e extracurriculares executadas a partir da aplicação da metodologia Problem-Based Learning (PBL), uma metodologia ativa que adaptamos para ser aplicada em ambiente on-line. Será apresentada a compreensão dos estudantes a respeito de aspectos essenciais na experiência realizada, como aqueles relacionados à construção do problema, infraestrutura da execução e competências e habilidades desenvolvidas. 


\section{Introdução}

Com a pandemia causada pelo coronavírus, o andamento das aulas foi afetado drasticamente. Com 139 milhões de pessoas contaminadas em todo mundo [WHO 2021] e poucas certezas relacionadas ao fim da crise global, as aulas presenciais se mantêm suspensas em muitos países, como no Brasil. Muitas instituições de ensino precisaram se adaptar ao ensino remoto para dar continuidade à oferta de ensino. Particularmente o Instituto Federal da Bahia estabeleceu as normas acadêmicas e provisórias para as Atividades Educacionais Não Presenciais Emergenciais (AENPE) durante o período de suspensão das atividades presenciais através da Resolução CONSUP/IFBA n ${ }^{\circ} 19$, de 24 de agosto de 2020 [da Bahia 2020a], atualizada, posteriormente, pela Resolução CONSUP/IFBA n ${ }^{\circ}$ 30, de 23 de Dezembro de 2020 [da Bahia 2020b].

Dada a possibilidade de continuidade das aulas durante a pandemia da Covid-19, porém agora de maneira remota através das AENPE, os docentes autores deste trabalho consideraram a aplicação da metodologia PBL (Problem Based Learning) como uma estratégia para tornar a aprendizagem dos seus estudantes mais eficiente quando comparada com uma abordagem mais tradicional. Isto porque, sendo uma metodologia ativa, um dos objetivos do PBL é tornar o aluno centro do processo de aprendizagem e o professor um facilitador, que incentiva o estudante a ter autonomia na busca pelo conhecimento.

A experiência exigiu que o PBL fosse adaptado para o ambiente online, já que comumente é utilizado na modalidade presencial de ensino. Nesse sentido, o suporte de ferramentas digitais é essencial para viabilizar que as etapas de execução da metodologia sejam realizadas, como, por exemplo, a ocorrência dos encontros online entre professor e alunos.

A aplicação do PBL ocorreu em semestre extraordinário (2020.4) em dois componentes: um curricular - a disciplina Arquitetura de Computadores e Software Básico, ofertada para os estudantes do curso Tecnólogo em Redes de Computadores no campus de Santo Antônio de Jesus, e o outro extracurricular - o curso livre Desenvolvimento de Games com Godot Engine, ofertado para estudantes do curso de Bacharelado em Sistemas de Informação do campus Feira de Santana. O suporte tecnológico para implementação do PBL online se deu principalmente através do Microsoft Teams, um ambiente virtual com licença educacional adquirida pelo IFBA.

Para possibilitar análise dos impactos da aplicação do PBL online no processo de ensino aprendizagem, os estudantes opinaram sobre a experiência realizada, respondendo a um questionário cujas questões se relacionam com aspectos essenciais da metodologia: a construção do problema, ambiente virtual de aprendizagem, competências e habilidades, tutor e conteúdo teórico abordado pelos docentes dos componentes durante momentos de aulas expositivas. Além disso, dados relacionados às condições sócio-econômicas e emocionais dos alunos, bem como condições de acesso à Internet também foram coletados em uma tentativa de compreender como questões que não são controladas pela instituição de ensino poderiam interferir na realização dos experimentos. Este trabalho se limita, porém, a apresentar a percepção dos estudantes quanto a aspectos específicos: problema tutorial, AVA e competências e habilidades. 


\section{PBL online no IFBA}

O processo de ensino-aprendizagem é um campo de estudo bastante rico e complexo. Dentre os diferentes modelos de ensino-aprendizagem existentes, destacamos: memorísticas, compreensivas, significativas e criativas [De Miranda 2016]. Particularmente nesta última, o aluno é visto como sujeito ativo, crítico e reflexivo, capaz de produzir algo novo baseado nas informações dadas [De Miranda 2016]. A partir do modelo de Edgar Dale, conhecido como Pirâmide da Aprendizagem [Dale 1969], observou-se que as estratégias ativas podem apresentar resultados mais consistentes de ensino-aprendizagem.

Uma metodologia ativa é um modelo de ensino centrado no aluno que tem como objetivo principal colocá-lo como agente ativo, crítico e reflexivo do seu processo de aprendizagem [De Miranda 2016]. Estas metodologias incentivam que os alunos leiam, escrevam, discutam e, até, busquem a resolução de situações e problemas do cotidiano [dos Santos et al. 2007]. Como exemplo de algumas dessas metodologias, tem-se o modelo da sala de aula invertida [Bergmann and Sams 2016], a gamificação [Fardo 2013] e o PBL (Problem-based Learning) [Delisle et al. 1997, dos Santos et al. 2007].

O PBL propõe que, a partir de informações iniciais, os educandos produzam seus próprios planos para resolver uma série de problemas [Delisle et al. 1997, dos Santos et al. 2007]. A dinâmica da execução do PBL é composta pelo: grupo tutorial, sessão tutorial, o problema, o tutor e o aluno. Em síntese:

- Grupo tutorial: representa a equipe responsável por discutir e propor a solução para um problema [Woods 1997].

- Sessão tutorial: consiste no ambiente em que ocorrem as discussões em busca da solução para o problema.

- Problema: descreve uma situação, ou cenário, do mundo real que possa promover o desenvolvimento dos estudos.

- Tutor: docente que tem a função de facilitador do aprendizado durante as discussões dos alunos nas sessões tutoriais. Este ator precisa garantir que a discussão entre os alunos seja uniforme, formular questões para nortear as discussões, entre outras ações [dos Santos et al. 2007].

- Alunos: atores que atuam na busca pelo conhecimento a fim de encontrar a solução para o problema proposto

Para aplicar o PBL nos componentes conduzidos pelos docentes (disciplina Arquitetura de Computadores e Software Básico e curso livre Desenvolvimento de Games com Godot Engine), os estudantes matriculados foram divididos em grupos tutoriais formados por 8 a 12 membros e, juntos, construíram e apresentaram soluções para problemas com temáticas específicas às áreas daqueles componentes. $\mathrm{O}$ ambiente digital que possibilitou o gerenciamento dos processos relacionados à execução da metodologia foi o software Microsoft Teams, cuja licença educacional foi adquirida pelo IFBA após interrupção das suas atividades acadêmicas presenciais.

O Microsoft Teams é uma ferramenta tecnológica digital de informação e comunicação que foi projetada para, também, funcionar como um Ambiente Virtual de Aprendizagem (AVA). O Teams reúne, em uma só plataforma, diferentes recursos tecnológicos que permitem aos professores criarem ambientes de aprendizagem personalizados e dinâmicos [Microsoft 2021]. Dentre os recursos disponíveis, destaca-se: 
criação de salas de aula virtuais; organização das equipes em canais; armazenamento e disponibilização de material didático; ferramenta para reunião com opção para gravação, interações e compartilhamento de conteúdo; chats privados e em grupo; mural coletivo; entre outros.

Através dos recursos tecnológicos do Teams foi possível transpor a estrutura presencial do PBL para o remoto. A execução da metodologia PBL exige que a turma seja dividida em grupos tutoriais e que cada grupo tenha seus respectivos momentos síncronos para discussão (sessões tutoriais) e compartilhamento de materiais inerentes à resolução do problema. Além disso, exige que os estudantes assumam papéis durante as reuniões.

No contexto das AENPE do IFBA, para a execução do PBL na disciplina de Arquitetura de Computadores e Software Básico e no curso livre Desenvolvimento de Games com Godot Engine, foi estruturado um ambiente virtual no Teams. Para cada atividade de ensino foi criada uma Equipe e os estudantes foram adicionados. A Equipe foi organizada em canais públicos, nos quais ocorriam as atividades síncronas e assíncronas comum a todos e em canais privados, onde foram organizados os grupos tutoriais. Devido à estrutura privada, somente os alunos inscritos em cada canal tinham acesso às atividades desenvolvidas dentro destes, sendo assim, garantindo a privacidade dos materiais e discussões ocorridas em cada grupo tutorial.

As sessões tutoriais foram executadas nos respectivos canais privados através do recurso de reuniões do Teams. Durante as sessões, os coordenadores puderam utilizar as ferramentas de "levantar a mão", ativar e desativar mudo, entre outras, para garantir a organização da discussão. O secretário de quadro utilizou o recurso de compartilhamento de uma tela do PowerPoint para que os demais estudantes pudessem acompanhar as anotações de quadro da sessão. O secretário de mesa pôde utilizar o recurso, também integrado ao Teams, do Word e a possibilidade de disponibilizar o relatório gerado como material didático no canal privado. Como forma de registro, todas as sessões tutoriais foram gravadas e disponibilizadas aos estudantes.

\section{Instrumento Diagnóstico}

Após finalização da execução das etapas metodológicas do PBL, com entrega de soluções dos estudantes e feedback dos tutores sobre as soluções apresentadas, disponibilizou-se aos estudantes um instrumento diagnóstico a ser preenchido por eles para obtenção das suas opiniões a respeito da experiência vivenciada. A Tablela 1 apresenta o instrumento diagnóstico proposto. Em sua construção, 37 afirmações foram elaboradas para 5 categorias diferentes: 1 - Ambiente Virtual de Aprendizagem, 2 - Problema, 3 - Tutor, 4 Conteúdo Teórico e 5 - Competências e Habilidades. As respostas ao questionário se baseiam no formato da escala Likert de maneira que os estudantes precisam especificar seu nível de concordância para as afirmações apresentadas, escolhendo uma das opções: Discordo Totalmente, Discordo, Não estou decidido, Concordo, Concordo Totalmente.

\section{Percepção dos estudantes}

Após a finalização dos cursos, o docente responsável por cada turma solicitou aos alunos que respondessem o questionário online. Ao todo, 22 estudantes responderam ao questionário sobre a aplicação do PBL. Destes, 8 foram alunos do curso Desenvolvimento de Games com Godot Engine e 14 da disciplina Arquitetura de Computadores e Software 
Tabela 1. Itens do Instrumento Diagnóstico

\begin{tabular}{|c|c|}
\hline Item & Categorias \\
\hline $\begin{array}{l}\text { O Ambiente Virtual de Aprendizagem (Microsoft Teams) utilizado na AENPE: } \\
\text { - Satisfez minhas expectativas } \\
\text { - Foi de fácil utilização } \\
\text { - Proporcionou uma boa aprendizagem. } \\
\text { - Viabilizou a execução da metodologia PBL. } \\
\text { - Me estimulou a aprender } \\
\text { - Me estimulou a participar das atividades } \\
\text { - Possibilitou a execução dos papéis nas sessões tutoriais }\end{array}$ & 1 \\
\hline $\begin{array}{l}\text { Os seguintes recursos do Microsoft Teams foram adequados para a execução da } \\
\text { metodologia ativa PBL: } \\
\text { - Organização da Equipe em canais } \\
\text { - Aba de Postagens dos canais } \\
\text { - Chat Privado. } \\
\text { - Reunião. } \\
\text { - Chat da reunião } \\
\text { - PowerPoint para exibição do quadro } \\
\text { - Disponibilização de Arquivos } \\
\text { - Disponibilização de vídeos gravados }\end{array}$ & 1 \\
\hline $\begin{array}{l}\text { O Problema: } \\
\text { - Foi bem elaborado e de fácil compreensão } \\
\text { - Utilizou temas envolvendo situações contextualizadas e atuais } \\
\text { - Exigiu a utilização de conceitos fundamentais relacionados à AENPE } \\
\text { - Estimulou as discussões em grupo }\end{array}$ & 2 \\
\hline $\begin{array}{l}\text { O Tutor: } \\
\text { - Estimulou o desenvolvimento do raciocínio dos estudantes } \\
\text { - Estimulou a participação ativa de todos os estudantes no grupo tutorial } \\
\text { - Atuou como mediador possibilitando que o grupo encontre } \\
\text { sua própria solução para o problema } \\
\text { - Forneceu feedback sobre o produto (código, relatório, apresentação, etc.). } \\
\text { - Forneceu feedback sobre o desempenho individual e do grupo. }\end{array}$ & 3 \\
\hline $\begin{array}{l}\text { O conteúdo teórico, abordado nos encontros síncronos e atividades assíncronas: } \\
\text { - Foi bem elaborado } \\
\text { - Foi bem conduzido } \\
\text { - Me auxiliou na resolução do problema }\end{array}$ & 4 \\
\hline $\begin{array}{l}\text { Sinto que desenvolvi a competência/habilidade: } \\
\text { - Trabalho em Equipe } \\
\text { - Liderança } \\
\text { - Comunicação interpessoal } \\
\text { - Expressão oral e escrita } \\
\text { - Autonomia em buscar o conhecimento } \\
\text { - Respeito por opiniões diversas } \\
\text { - Ética } \\
\text { - Demonstrar interesse e responsabilidade em auxiliar colegas } \\
\text { - Apresentar ideias de forma lógica, concisa e ordenada } \\
\text { - Domínio na utilização de ferramentas digitais }\end{array}$ & 5 \\
\hline
\end{tabular}


Básico, correspondendo a $100 \%$ dos alunos que frequentaram o primeiro curso e $61 \%$ dos que frequentaram o segundo curso.

Em um questionamento inicial do formulário, foi realizada a consulta sobre experiências anteriores com o método PBL. $72,7 \%$ dos alunos afirmaram que não tiveram experiências anteriores com o PBL e 27,3\% afirmaram que sim. Dessa forma, para a maioria dos alunos, o PBL apresentou-se como uma experiência nova no processo de ensino e aprendizagem.

Do ponto de vista da percepção do aluno sobre a execução do PBL de forma remota em Atividades Educacionais Não Presenciais Emergenciais no IFBA, este artigo aborda o Ambiente Virtual de Aprendizagem, o Problema e as Habilidades e Competências desenvolvidas.

Na categoria sobre o Microsoft Teams foram elaboradas afirmações sobre o AVA de forma a obter o feedback dos alunos sobre a eficácia da ferramenta na aplicação do PBL de forma remota. Na Figura 1 pode-se observar que cerca de $73 \%$ dos alunos concordaram ou concordaram totalmente que o Teams viabilizou a execução da metodologia PBL. Com isso, atesta-se que para os alunos o Teams garantiu, de forma satisfatória, a execução das ações didático pedagógicas inerentes ao PBL.

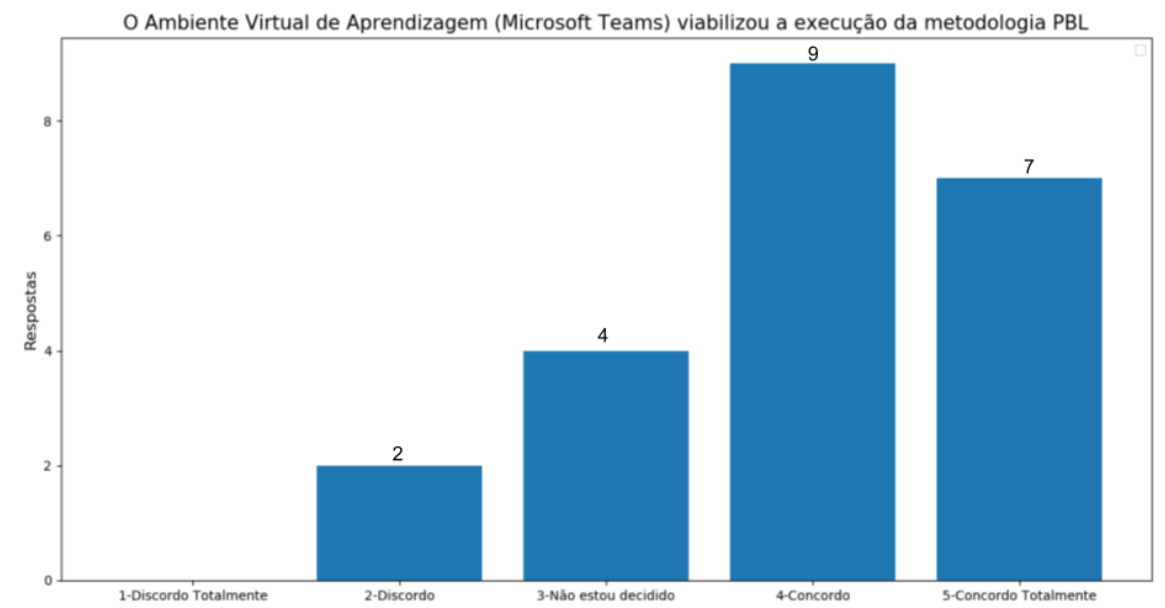

Figura 1. Gráfico sobre a viabilização da execução do PBL no Microsoft Teams

Além da percepção geral sobre a execução do PBL, foi levantado também a opinião dos estudantes sobre a execução dos papéis nas sessões tutoriais através do Teams. A Figura 2 apresenta que 86,36\% das respostas concordaram ou concordaram totalmente que o Teams possibilitou o desenvolvimento das atividades de coordenação, secretário de mesa e de quadro.

O processo de ensino e aprendizagem da metodologia PBL envolve a discussão e a resolução de problemas que descrevem situações do mundo real. Ao final do processo de aplicação do PBL, como forma de avaliar a percepção dos alunos sobre o problema, foram colocadas afirmações no questionário sobre a compreensão e elaboração do problema, a contextualização da situação descrita com a atualidade, os conceitos fundamentais relacionados à AENPE e o estímulo às discussões do grupo. 


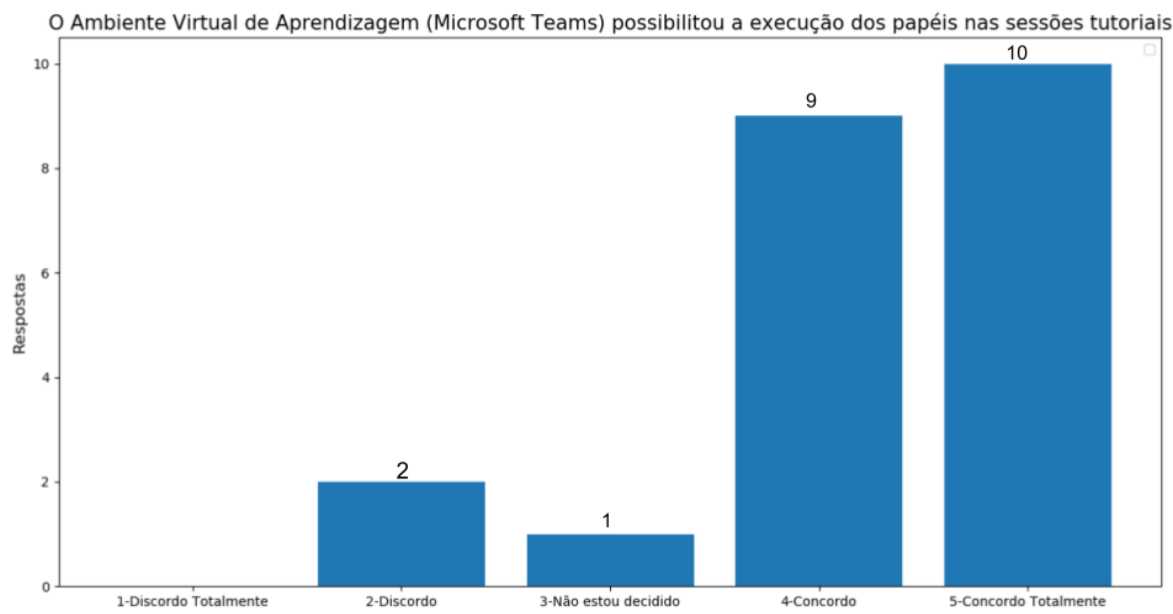

Figura 2. Gráfico sobre a viabilização da execução dos papeis nas sessões tutoriais através do Teams

A Figura 3 ilustra o gráfico construído a partir das respostas dos alunos sobre as afirmações relacionadas ao problema. Observa-se que, em todos as afirmações, a maioria dos alunos concordaram ou concordaram totalmente. Com isso, pode-se extrair que os estudantes envolvidos nas AENPE consideraram que o problema foi de fácil compreensão, bem elaborado, abordou temas contextualizados e atuais, envolveu conceitos fundamentais à AENPE e também estimulou a discussão em grupo.

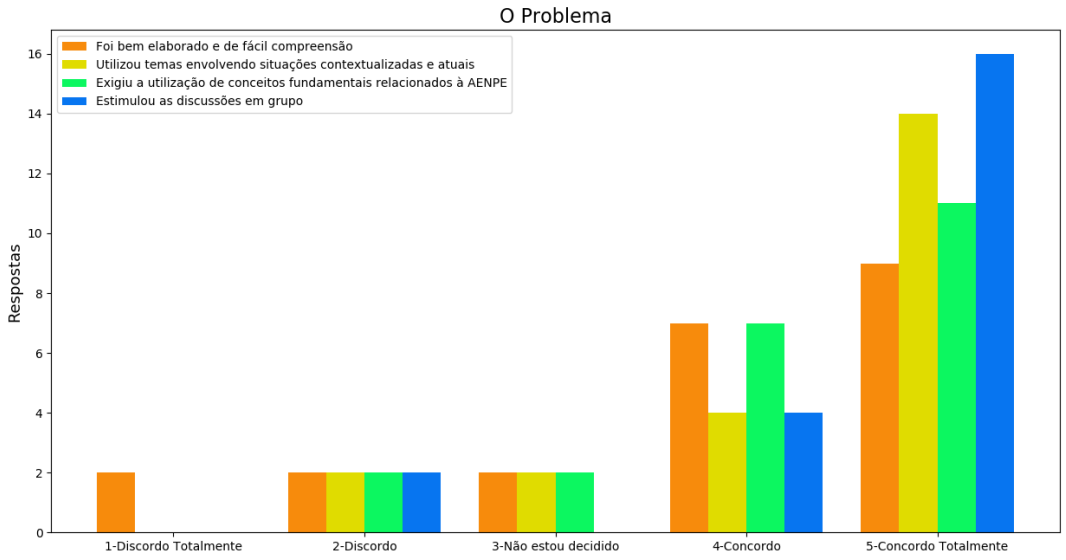

Figura 3. Gráfico das percepções sobre o problema

Em outra categoria do questionário, foram colocadas afirmações sobre competências e habilidades desenvolvidas pelos estudantes durante a execução do PBL de forma remota. O gráfico apresentado na Figuras 4 destaca o feedback dos alunos sobre as seguintes habilidades e competências: Trabalho em Equipe, Autonomia em buscar o conhecimento, Demonstrar interesse e responsabilidade em auxiliar colegas e Apresentar ideias de forma lógica, concisa e ordenada. Em todas as habilidades e competências destacadas no gráfico, observa-se que a maioria dos alunos concordaram ou concordaram totalmente que as mesmas foram desenvolvidas. 


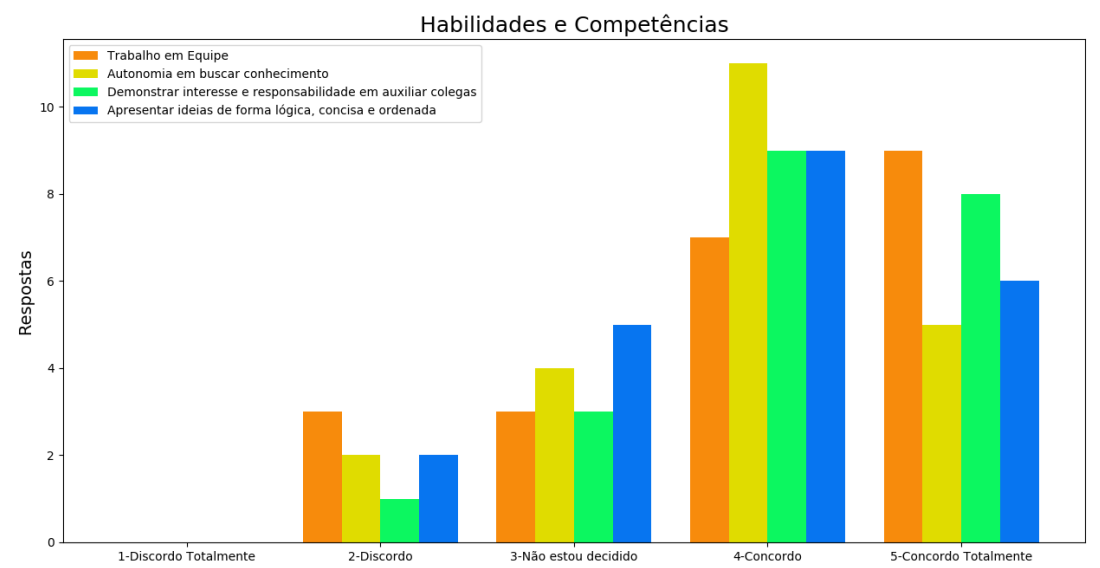

Figura 4. Gráfico sobre a Habilidade/Competência de Trabalho em Equipe

Apesar das boas avaliações, alguns estudantes evidenciaram problemas, entre eles se observa a dificuldade com acesso a internet, um dos grandes desafios na aplicação da metodologia de forma remota. O gráfico apresentado na Figura 5 mostra que cerca de $23 \%$ dos alunos destacaram a dificuldade com o acesso a internet.

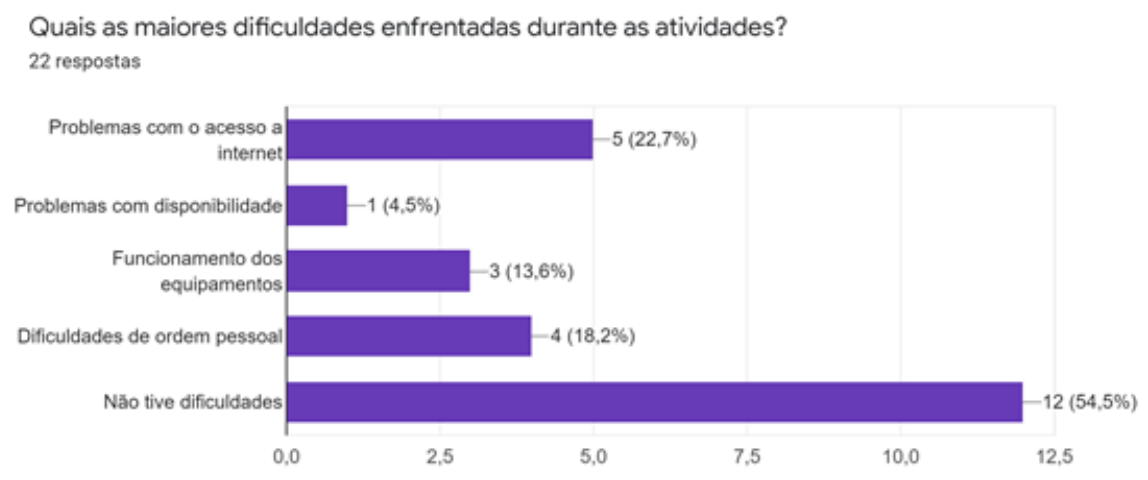

Figura 5. Gráfico sobre as maiores dificuldades enfrentadas

Um dos maiores desafios para o ensino remoto é a dificuldade de acesso a internet por parte dos alunos. Neste contexto, foi analisado o feedback dos alunos que atestaram problemas de internet sobre o uso do Teams. De forma esperada, analisando o gráfico da Figura 6 a maioria atestou não estar decidida se o Teams viabilizou a execução da metodologia. A qualidade na internet afeta, diretamente, a experiência do usuário com as ferramentas digitais.

\section{Conclusão e Trabalhos Futuros}

A pandemia da COVID-19 tem se apresentado como um grande desafio para diferentes setores da sociedade. Estratégias para conter a transmissão do novo coronavírus resultaram em distanciamento social que, no contexto educacional, resultou na remodelagem dos processos de ensino com adaptação das aulas para ambiente remoto. 


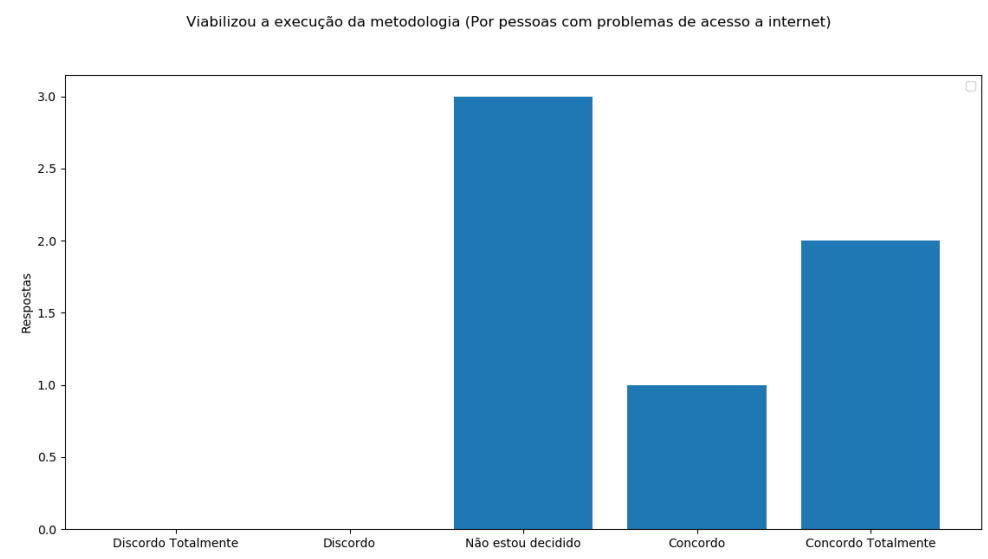

Figura 6. Pessoas com problemas de acesso a internet e a utilização do Teams

A necessidade de inovar na prática docente para gerar maior engajamento dos estudantes nas atividades acadêmicas e garantir a manutenção da qualidade no processo de ensino-aprendizagem resultou na aplicação de metodologia ativa, especificamente o PBL, em componentes ofertados a estudantes de curso superior na área da computação no Instituto Federal da Bahia. Foi necessário, para isso, adaptar o PBL para um ambiente totalmente remoto e o suporte do Ambiente Microsoft Teams foi importante para implementar as características essenciais da metodologia.

Este artigo se limitou a apresentar a avaliação do impacto da utilização do PBL do ponto de vista de alguns aspectos, como o Ambiente Virtual de Aprendizagem, o Problema e as Habilidades e Competências desenvolvidas. A partir das análises dos aspectos sobre o AVA, observou-se que os alunos tiveram uma experiência exitosa com o Microsoft Teams, atestando que os recursos tecnológicos disponíveis contribuíram para a execução do PBL.

Sobre o problema apresentado aos alunos, observou-se que a maioria deles concordou que a situação problema apresentada contribuiu para o desenvolvimento dos estudos, abrangendo conceitos fundamentais relacionados à AENPE. Outro aspecto também levantado neste trabalho foram as habilidades e competências desenvolvidas pelos alunos durante a experiência com o PBL remoto. Em todos os critérios analisados, a maioria dos alunos concordaram que as habilidades e competências foram desenvolvidas.

Como trabalho futuro, as demais percepções dos estudantes participantes do estudo, sobretudo sobre o papel do tutor e o conteúdo teórico abordado pelo docente, serão analisadas e discutidas. Além destas percepções, serão relacionadas também as de que forma as condições sócio-econômicas e emocionais dos alunos durante a pandemia influenciaram o seu desempenho nas atividades realizadas, já que estes dados já foram coletados. As análises sobre estes pontos precisam ser construídas e discutidas de forma a revelar as limitações da proposta e gerar melhorias que possam atrair o aluno para sala de aula virtual e resultar no alcance dos objetivos previstos para o discente quando do planejamento dos componentes.

Pretende-se, ainda, ampliar o escopo do trabalho e aplicar a metodologia em mais turmas de maneira a averiguar se a satisfação dos alunos é novamente alcançada. A al- 
ternância do ambiente virtual de aprendizagem também pode ser realizada no sentido de verificar quais AVAs são mais indicados para a aplicação do PBL online.

\section{Agradecimentos}

Os autores agradecem ao Instituto Federal da Bahia (IFBA) por seu apoio financeiro.

\section{Referências}

Bergmann, J. and Sams, A. (2016). Sala De Aula Invertida: Uma Metodologia Ativa de Aprendizagem. LTC.

da Bahia, I. F. (2020a). Res. nº 19 - revoga res. 18 e regulamenta a implementação aenpe nos cursos do ifba. https://portal.ifba.edu.br/institucional/ consup/resolucoes-2020. (Accessed on 04/16/2021).

da Bahia, I. F. (2020b). Res. no 30 - altera a res. 19_2020, que regulamenta as aenpe nos cursos do ifba. https://portal.ifba.edu.br/institucional/ consup/resolucoes-2020/res-no-30-altera-a-res-19_ 2020-que-regulamenta-as-aenpe-nos-cursos-do-ifba.pdf/ view. (Accessed on 04/16/2021).

Dale, E. (1969). Audiovisual Methods in Teaching. Dryden Press.

De Miranda, S. (2016). Estrategias Didaticas Para Aulas Criativas. PAPIRUS.

Delisle, R., for Supervision, A., and Development, C. (1997). How to Use Problem-based Learning in the Classroom. ASCD.

dos Santos, D. M. B., Pinto, G., Sena, C. P. P., Bertoni, F. C., and Bittencourt, R. A. (2007). Aplicação do método de aprendizagem baseada em problemas no curso de engenharia da computação da universidade estadual de feira de santana. In Congresso Brasileiro de Educação em Engenharia-COBENGE.

Fardo, M. L. (2013). A gamificação aplicada em ambientes de aprendizagem. RENOTERevista Novas Tecnologias na Educação, 11(1).

Microsoft (2021). Microsoft teams - aplicativo de chat interno - trabalho remoto. https://www.microsoft.com/pt-br/microsoft-teams/ group-chat-software. (Accessed on 04/13/2021).

WHO (2021). Who coronavirus (covid-19) dashboard - who coronavirus (covid-19) dashboard with vaccination data. https://covid19. who.int/. (Accessed on 04/16/2021).

Woods, D. (1997). Problem-based Learning: Resources to Gain the Most from PBL. D.R. Woods. 